\title{
Gross Motor Function Outcome After Intensive Rehabilitation in Children With Bilateral Spastic Cerebral Palsy
}

\author{
Seung Hoon Lee, MD, Jae Sun Shim, MD, Kiyoung Kim, MD, \\ Jinkyoo Moon, MD, MinYoung Kim, MD, PhD
}

Department of Rehabilitation Medicine, CHA Bundang Medical Center, CHA University, Seongnam, Korea

Objective To compare gross motor function outcomes in children with moderate to severe degrees of bilateral spastic cerebral palsy (CP) who received either intensive inpatient rehabilitation or intermittent rehabilitation on an outpatient basis.

Methods A non-biased retrospective chart review was done for patients diagnosed with bilateral spastic CP who received rehabilitation therapy. The intensive rehabilitation group (inpatient group) agreed to be hospitalized to receive 22 sessions of physical and occupational therapy per week for 1 month. The intermittent rehabilitation group (outpatient group) received four sessions of physical and occupational therapy per week for 3 months in an outpatient setting. Changes in the total score on the Gross Motor Function Measure (GMFM) between baseline and the follow-up period were analyzed.

Results Both groups showed significant improvements in total GMFM scores at the follow-up assessment compared to that at baseline ( $\mathrm{p}=0.000$ for inpatient group, $\mathrm{p}=0.001$ for outpatient group). The increase in mean total GMFM score after 1 month was significantly greater in the inpatient group than that in the outpatient group ( $\mathrm{p}=0.020$ ). Higher increase in GMFM score was observed in younger subjects as revealed by the negative correlation between age and the increase in GMFM score after 1 month ( $p=0.002, r=-0.460)$.

Conclusion Intensive inpatient rehabilitation therapy for patients with bilateral spastic CP of moderate to severe degree was more effective for improving gross motor function than intermittent rehabilitation therapy on an outpatient basis.

Keywords Intensive rehabilitation, Cerebral palsy, The Gross Motor Function Measure, Age

Received November 6, 2014; Accepted April 13, 2015

Corresponding author: MinYoung Kim

Department of Rehabilitation Medicine, CHA Bundang Medical Center, CHA University, 59 Yatap-ro, Bundang-gu, Seongnam 463-712, Korea Tel: +82-31-780-6281, Fax: +82-31-780-3449, E-mail: kmin@cha.ac.kr

(c) This is an open-access article distributed under the terms of the Creative Commons Attribution Non-Commercial License (http://creativecommons. org/licenses/by-nc/4.0) which permits unrestricted noncommercial use, distribution, and reproduction in any medium, provided the original work is properly cited.

Copyright $\odot 2015$ by Korean Academy of Rehabilitation Medicine

\section{INTRODUCTION}

Impaired development of gross motor function is a major characteristic of cerebral palsy (CP) [1]. Gross motor function, such as the ability to walk, is closely related to other problems in patients with $\mathrm{CP}$, such as cognitive, visual, and auditory impairments, and is considered an indicator of overall prognosis [2]. 
Appropriate gross motor function assessments are important when treating patients with CP. The Gross Motor Function Measure (GMFM) is a validated tool to assess motor function in children with CP. It measures gross motor function during lying and rolling, crawling and kneeling, sitting, standing, and walk-run-jump activities with a scale of 0-100 [3]. Reliability of the GMFM has been tested in many studies and the measure is appropriate for evaluating changes in gross motor function in children with CP [3-7]. The five levels of the Gross Motor Function Classification System (GMFCS) have been widely employed in children $<12$ years of age with $\mathrm{CP}$ and focuses on sitting and walking ability. The GMFCS classification levels are determined based on functional limitations, dependence on assistive devices or wheelchairs, and quality of movement $[8,9]$. The validity, reliability, and stability of the GMFCS have been tested in previous studies [6-11].

Among the several types of $\mathrm{CP}$, bilateral spastic $\mathrm{CP}$ is the most common, accounting for about $55 \%$ of the CP population. The overall incidence rate of bilateral spastic CP per 1,000 live births is 1.16 [12]. Periventricular leukomalacia (PVL) and parasagittal subcortical injury are the main magnetic resonance imaging (MRI)-detected pa- thologies of bilateral spastic CP [13], and diffuse bilateral insult to the brain, such as hypoxic ischemic encephalopathy (HIE) can cause quadriplegic $\mathrm{CP}$, a severe form of bilateral spastic CP [14].

Rehabilitation therapy has been used to improve functional outcomes of patients with CP. Quantitative intensive rehabilitation with neurodevelopmental and goal-directed treatments has shown stronger effects on gross motor function of children with $\mathrm{CP}$ than intermittent treatments $[15,16]$. However, the intensive physical therapy required for the $\mathrm{CP}$ population may become an enormous social burden. Therefore, a proper evaluation of the therapeutic benefit to establish appropriate indications is essential.

In this study, we compared changes in the GMFM score in children with moderate to severe bilateral spastic CP who received either intensive rehabilitation on hospitalization or intermittent rehabilitation on an outpatient basis.

\section{MATERIALS AND METHODS}

A non-biased retrospective chart review was done for patients with bilateral spastic CP who received physical

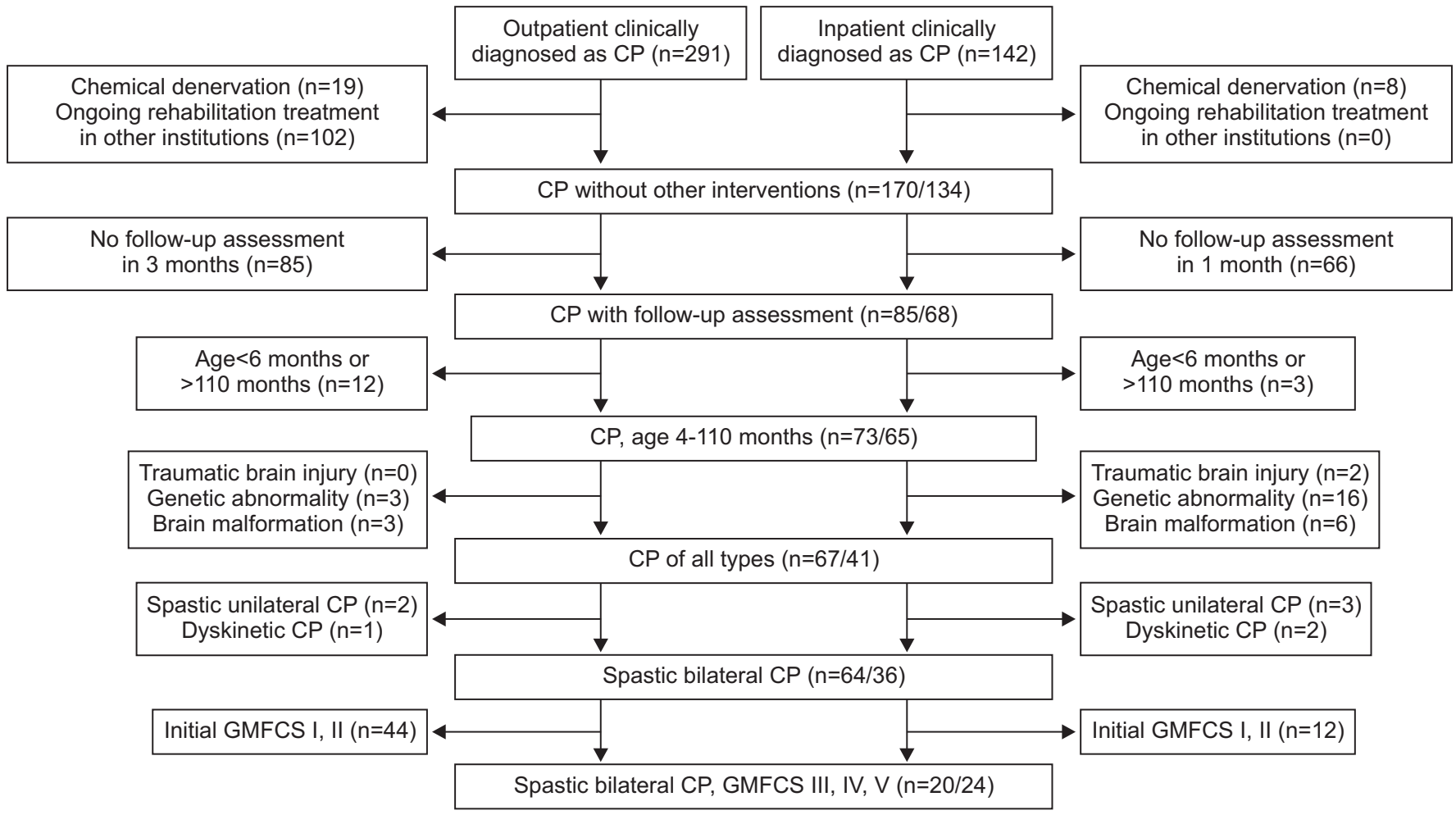

Fig. 1. Flow diagram of the study participants. CP, cerebral palsy; GMFCS, Gross Motor Function Classification Scale. 
therapy at CHA Hospital from January 2010 to January 2014. Those who were diagnosed with moderate to severe degree of CP during this period at this institution were primarily recommended for admission and inpatient rehabilitation. Most of the patients who disagreed to receive intermittent rehabilitation therapy at hospital admission were treated on an outpatient basis.

Inclusion criteria were 1) children aged from 6 months to 10 years, (2) clinically diagnosed with bilateral spastic $\mathrm{CP}, 3)$ GMFCS levels III-V, and 4) complete medical records of the GMFM assessment available for the initial and follow-ups evaluations at 1 month for the inpatient group and 3 months for the outpatient group. Exclusion criteria were 1) traumatic brain injury, 2) genetic abnormality or brain malformation, 3 ) history of surgery or cell therapy, 4) patients who received ongoing rehabilitation treatment at other institutions, and 5) any other treatments that may influence gross motor function including botulinum toxin, and chemical denervation treatment using alcohol or phenol within 6 months prior to recruitment and during rehabilitation. The clinical diagnosis of CP was made according to abnormal of physical examination findings, including muscle tonus, posture and movement pattern, and significant motor development delay as indicated by the Bayley Scale of Infant Development, 2nd edition. Medical history and brain images were also collected for the diagnosis. Consequently, 44 patients (24 in the inpatient group and 20 in the outpatient group) were enrolled (Fig. 1).

The intensive rehabilitation group (inpatient group) agreed to be hospitalized to receive 22 sessions of physical and occupational therapy per week (11 sessions per therapy), each session lasting 30 minutes for 4 weeks. The intermittent rehabilitation group (outpatient group) received four sessions of physical and occupational therapy per week (two sessions per therapy) for 3 months in an outpatient setting. Other conditions, including session duration and treatment strategy and technique provided were identical to those in the inpatient group.

Both groups received rehabilitation treatment based on a neurodevelopmental treatment combined with additional goal-directed therapy. The following principles were applied for physical therapy. 1) Individual treatment goals were established based on the GMFM items that were performed poorly by patients at the initial assessment. 2) Children with high physical function prac- ticed their tasks or related activities of daily living, such as 'sitting on a bench with arms and feet free.' 3) Children with poor physical function focused on improving either separate movements or the quality of movements, such as 'trunk and pelvic control or pelvic tilting training.'

The following principles were applied for occupational therapy. 1) Individual treatment goals were set according to the results of the Bayley Scale of Infant Development 2nd edition, the Beery-Buktenica Developmental Test of Visual-Motor Integration 5th edition, the Wee Functional Independence Measure for Children, the Sensory profile-3, and the Quality of Upper Extremity Skills Test. 2) An occupational therapist performed one-on-one training for problems with cognition, perception, hand function, activities of daily living, and sensory in order of priority for each patient. 3) Play therapy with skills inspiring motivation was the main principle of all treatment sessions.

Changes in the total GMFM-88 scores between baseline and follow-up period at 1 month for the inpatient group and 3 months for the outpatient group were calculated and analyzed with the paired t-test. The increases in total GMFM-88 score in the outpatient group was adjusted by time of 1 month to compare improvements in gross motor function between the two groups. The results were analyzed using the independent t-test. The MannWhitney test was used to compare the 1-month outcomes

Table 1. Baseline patient characteristics

\begin{tabular}{lcc}
\hline \multicolumn{1}{c}{ Characteristic } & $\begin{array}{c}\text { Outpatient } \\
\text { group (n=20) }\end{array}$ & $\begin{array}{c}\text { Inpatient } \\
\text { group (n=24) }\end{array}$ \\
\hline Sex (male:female) & $18: 2$ & $15: 9$ \\
Mean age (mo) & $27.6 \pm 21.5$ & $28.3 \pm 21.2$ \\
Gestational age (wk) & $32.9 \pm 4.7$ & $34.4 \pm 5.4$ \\
\hline Birth weight (g) & $2,029 \pm 947$ & $2,199 \pm 881$ \\
\hline Initial GMFM total score & $32.1 \pm 0.8$ & $24.6 \pm 19.9$ \\
Initial GMFCS level & & \\
\hline III & 10 & 7 \\
\hline IV & 5 & 7 \\
\hline V & 5 & 10 \\
\hline
\end{tabular}

Values are presented as mean \pm standard deviation or number.

No significant differences were observed between the two groups.

GMFCS, Gross Motor Function Classification Scale; GMFM, Gross Motor Function Measure. 
Table 2. Mean increase in total GMFM score after 1 month

\begin{tabular}{lccccl}
\hline Initial GMFCS level & No. of outpatient & Outpatient group & No. of inpatient & Inpatient group & p-value \\
\hline III & $10(8)$ & $2.86 \pm 2.66(2.06 \pm 2.30)$ & $7(6)$ & $7.10 \pm 4.81(7.36 \pm 5.22)$ & $0.064\left(0.028^{*}\right)$ \\
\hline IV & $5(3)$ & $2.88 \pm 1.98(1.53 \pm 0.99)$ & $7(4)$ & $6.85 \pm 5.61(5.70 \pm 5.43)$ & $0.167(0.077)$ \\
V & $5(4)$ & $0.68 \pm 1.27(0.15 \pm 0.47)$ & $10(7)$ & $2.52 \pm 3.77(1.45 \pm 1.83)$ & $0.206(0.113)$ \\
Total & $20(15)$ & $2.32 \pm 2.34(1.44 \pm 1.88)$ & $24(17)$ & $5.12 \pm 4.99(4.54 \pm 4.78)$ & $0.020^{*}\left(0.022^{*}\right)$ \\
\hline
\end{tabular}

GMFCS, Gross Motor Function Classification Scale; GMFM, Gross Motor Function Measure.

Mann-Whitney test was used to compare each GMFCS level, and the independent t-test was used for all patients in the two groups.

Data in parentheses are sub-analysis with restricted age of 12 months to 10 years.

*Significant difference between groups, indicated by $\mathrm{p}<0.05$.

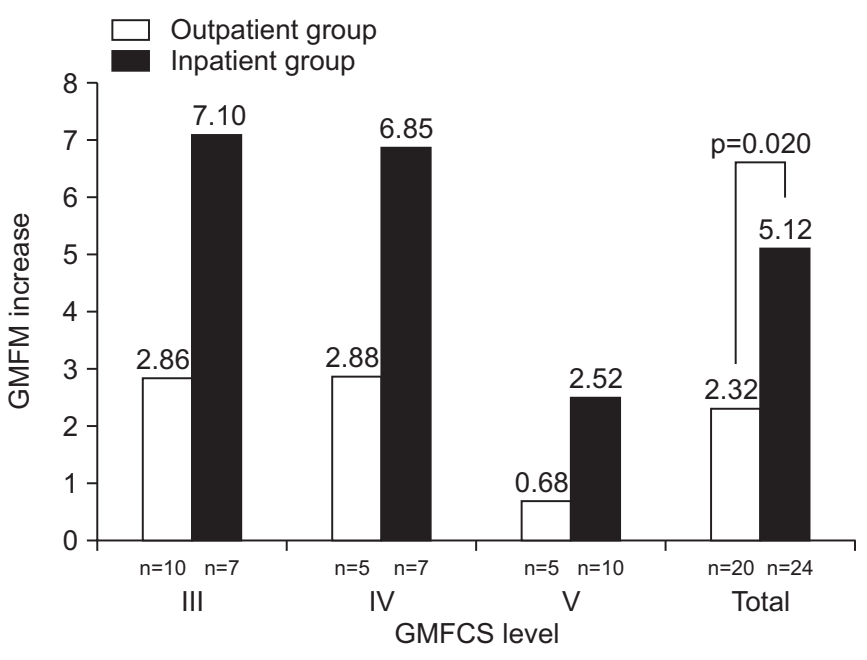

Fig. 2. Mean increase of total GMFM score after 1 month. GMFCS, Gross Motor Function Classification Scale; GMFM, Gross Motor Function Measure.

at each GMFCS level. Subgroup analyses for patients $>1$ year were conducted using the same statistical methods.

This study protocol was approved by the CHA Bundang Medical Center Institutional Review Board.

\section{RESULTS}

The study sample included 44 children with bilateral spastic CP; 20 patients in the outpatient group and 24 patients in the inpatient group. The follow-up durations were 30.5 \pm 3.7 days for the inpatient group and 101.2 \pm 27.9 days for the outpatient group. No significant differences were found in the baseline characteristics or the initial GMFM and GMFCS assessment measures between the groups (Table 1).

More than $70 \%$ of the participants showed PVL or HIE

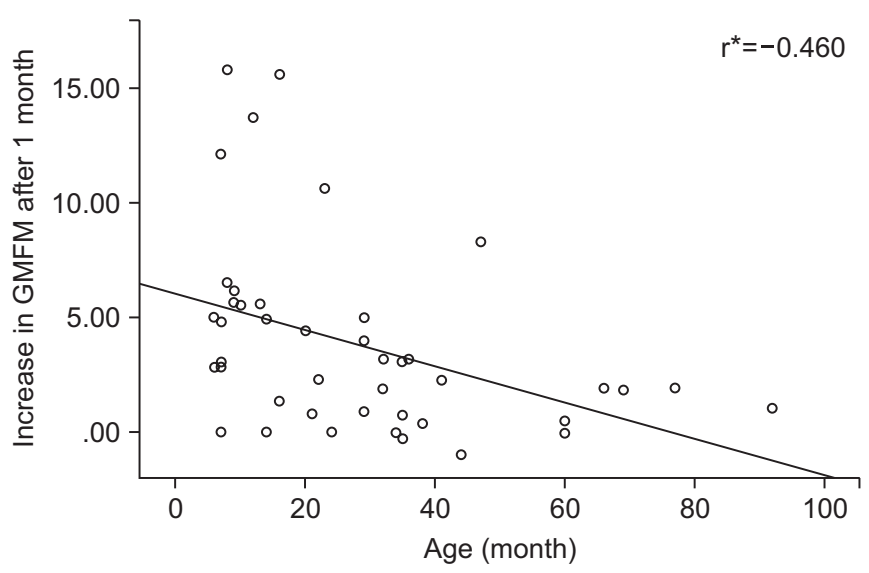

Fig. 3. Correlation between age and increase in the GMFM after 1 month. *Spearman correlation coefficient $(\mathrm{p}=0.002)$. GMFM, Gross Motor Function Measure.

on brain MRI; 12 PVLs and six HIEs in the outpatient group and 11 PVLs and seven HIEs in the inpatient group.

Both groups showed significant improvement on the total GMFM scores between baseline and follow-up assessments $(p=0.000$ for the inpatient group and $p=0.001$ for the outpatient group). The mean change in total GMFM score at 1 month was significantly greater in the inpatient group than that in the outpatient group $(\mathrm{p}=0.020)$. However, the analysis of each GMFCS level did not reveal any difference between the groups (Table 2, Fig. 2). The increase in GMFM scores after 1 month was negatively correlated with age $(\mathrm{p}=0.002, \mathrm{r}=-0.460)$ (Fig. 3).

A sub-analysis was conducted with age restricted to 12 months to 10 years for 15 outpatients (male 14, female 1) and 17 inpatients (male 10, female 7). Both groups showed significant improvements in the total GMFM scores between the baseline and follow-up assessments ( $p=0.001$ for the inpatient group and $\mathrm{p}=0.02$ for the out- 
patient group). The mean increase in total GMFM score at 1 month was significantly greater in the inpatient group than that in the outpatient group $(\mathrm{p}=0.022)$ (Table 2). Only participants with GMFCS level III showed a more significant improvement in total GMFM score in the inpatient group than those in the outpatient group $(p=0.028)$, while those at other GMFCS levels did not.

\section{DISCUSSION}

In this study, gross motor function in the inpatient group improved more than that in the outpatient group as reflected by the GMFM score changes. Considering the negative correlation between mean age and the increase in the GMFM score, younger age seemed to be a significant factor for a better gross motor function outcome with intensive treatment. The most prominent improvement was observed in patients in the inpatient group with GMFCS level III. Although statistically insignificant, GMFCS level IV also showed a similar characteristic, suggesting that therapy is more efficient for those who are capable of basic physical functions, such as creeping or sitting, than those who lacked these abilities.

The inpatient group had about 2.8 more points in the mean total GMFM score than that in the outpatient group after 1 month. This small change may lead to a large functional improvement when accumulated over several months or years. Children with CP have a limited time for gross motor improvement, and children with lower motor function tend to reach their limit more quickly [17]. For example, children at GMFCS level III are expected to reach $90 \%$ of their motor development potential at 3.7 years of the age, whereas those at GMFCS level I reach their potential at 4.8 years [17]. Thus, administering intensive physical therapy at a younger age may contribute to maximize physical functioning in children with $\mathrm{CP}$.

We used the GMFM-88 for the present study. Both the GMFM-88 and the GMFM-66 are valid tools to measure the outcome changes in gross motor function for children with CP undergoing interventions [18]. The GMFM66 is a recently developed version of the GMFM that uses Rasch analysis to reflect changes in gross motor function more accurately [4]. However, the GMFM-88 is more useful for evaluating changes in gross motor function of more severely impaired children with CP [19]. Moreover, the GMFM-88 provides an estimated total score goals for selected dimensions on which physical therapists need to focus. Thus, we used the GMFM-88 for follow-up evaluations and individual goal setting of the participants.

There were some limitations in this study. First, this was not a prospective study. The follow-up assessment time was different between the two groups because of limitations in current clinical practice; 1 month for the inpatient group and 3 months for the outpatient group. We corrected the GMFM changes of the outpatient group at 1 month under the assumption that the GMFM score increases linearly. However, the efficacy of intensive inpatient treatment could be different if hospitalization duration is extended. Thus, a subsequent study evaluating the effects of longer intensive treatment are necessary. Second, the GMFM and GMFCS may not be appropriate for infants $<1$ year of age, as they have not been validated for this age group. Thus, a sub-analysis with age restricted from 12 months to 10 years was conducted. The results revealed similar benefits from intensive therapy for those more than 1 year old. Further studies with a larger number of patients with various types of CP involving different ages and severities would verify the value of intensive rehabilitation.

In conclusion, intensive inpatient rehabilitation therapy for patients with bilateral spastic CP of moderate to severe degree was more effective in improving gross motor function than intermittent rehabilitation therapy on an outpatient basis. Particularly, the effect seemed to be greater in younger patients and in children at GMFCS level III. A prospective, controlled study with a larger number of patients with various types of $\mathrm{CP}$ involving different ages and severities will be helpful to establish the effectiveness and the exact indications for intensive rehabilitation for patients with CP.

\section{CONFLICT OF INTEREST}

No potential conflict of interest relevant to this article was reported.

\section{REFERENCES}

1. Baxter P, Morris C, Rosenbaum P, Paneth N, Leviton A, Goldstein $\mathrm{M}$, et al. The definition and classification of cerebral palsy. Dev Med Child Neurol 2007;49:1-44.

2. Beckung E, Hagberg G, Uldall P, Cans C; Surveillance 
of Cerebral Palsy in Europe. Probability of walking in children with cerebral palsy in Europe. Pediatrics 2008;121:e187-92.

3. Russell DJ, Rosenbaum PL, Cadman DT, Gowland C, Hardy S, Jarvis S. The gross motor function measure: a means to evaluate the effects of physical therapy. Dev Med Child Neurol 1989;31:341-52.

4. Russell DJ, Avery LM, Rosenbaum PL, Raina PS, Walter SD, Palisano RJ. Improved scaling of the gross motor function measure for children with cerebral palsy: evidence of reliability and validity. Phys Ther 2000;80:873-85.

5. Avery LM, Russell DJ, Raina PS, Walter SD, Rosenbaum PL. Rasch analysis of the Gross Motor Function Measure: validating the assumptions of the Rasch model to create an interval-level measure. Arch Phys Med Rehabil 2003;84:697-705.

6. Ko J, Kim M. Reliability and responsiveness of the gross motor function measure-88 in children with cerebral palsy. Phys Ther 2013;93:393-400.

7. Ko J, Kim M. Inter-rater Reliability of the K-GMFM-88 and the GMPM for children with cerebral palsy. Ann Rehabil Med 2012;36:233-9.

8. Palisano R, Rosenbaum P, Walter S, Russell D, Wood E, Galuppi B. Development and reliability of a system to classify gross motor function in children with cerebral palsy. Dev Med Child Neurol 1997;39:214-23.

9. Palisano RJ, Hanna SE, Rosenbaum PL, Russell DJ, Walter SD, Wood EP, et al. Validation of a model of gross motor function for children with cerebral palsy. Phys Ther 2000;80:974-85.

10. Wood E, Rosenbaum P. The gross motor function classification system for cerebral palsy: a study of reliability and stability over time. Dev Med Child Neurol
2000;42:292-6.

11. Palisano RJ, Cameron D, Rosenbaum PL, Walter SD, Russell D. Stability of the gross motor function classification system. Dev Med Child Neurol 2006;48:424-8.

12. Prevalence and characteristics of children with cerebral palsy in Europe. Dev Med Child Neurol 2002; 44:633-40.

13. Krageloh-Mann I, Petersen D, Hagberg G, Vollmer B, Hagberg B, Michaelis R. Bilateral spastic cerebral palsy: MRI pathology and origin. Analysis from a representative series of 56 cases. Dev Med Child Neurol 1995;37:379-97.

14. Nelson KB. Causative factors in cerebral palsy. Clin Obstet Gynecol 2008;51:749-62.

15. Tsorlakis N, Evaggelinou C, Grouios G, Tsorbatzoudis C. Effect of intensive neurodevelopmental treatment in gross motor function of children with cerebral palsy. Dev Med Child Neurol 2004;46:740-5.

16. Shamir M, Dickstein R, Tirosh E. Intensive intermittent physical therapy in infants with cerebral palsy: a randomized controlled pilot study. Isr Med Assoc J 2012;14:737-41.

17. Rosenbaum PL, Walter SD, Hanna SE, Palisano RJ, Russell DJ, Raina P, et al. Prognosis for gross motor function in cerebral palsy: creation of motor development curves. JAMA 2002;288:1357-63.

18. Alotaibi M, Long T, Kennedy E, Bavishi S. The efficacy of GMFM-88 and GMFM-66 to detect changes in gross motor function in children with cerebral palsy (CP): a literature review. Disabil Rehabil 2014;36:617-27.

19. Russell DJ, Rosenbaum PL, Avery LM, Lane M. Gross motor function measure (GMFM-66 and GMFM-88) user's manual. London: Mac Keith Press; 2002. 